\title{
MICROMECHANICAL CHARACTERIZATION OF ENERGY DISSIPATIVE MECHANISMS IN FRACTURE PROCESS ZONE
}

\author{
SUDAKSHINA DUTTA* AND J. M. CHANDRA KISHEN ${ }^{\dagger}$ \\ Indian Institute of Science \\ Department of Civil Engineering, Bangalore, INDIA \\ *e-mail:sdutta@civil.iisc.ernet.in \\ †e-mail:chandrak@civil.iisc.ernet.in
}

Key words: Debonding, Interface, Micromechanics, Softening

\begin{abstract}
Debonding of coarse aggregates from the surrounding matrix is considered as a major energy dissipative mechanism which affects the mechanical behavior of cementitious composites like concrete. In the present work, a micromechanical model has been developed to study the effect of debonding on the macroscopic behavior of concrete. Concrete is modeled as a two phase composite and a linear softening law has been used to characterize the interface between the phases. Numerical analysis is carried out to obtain the macroscopic stress-strain behavior under uniaxial tension. A parametric study is conducted to study how the various material properties of the individual phases affect the overall stress-strain behavior of concrete, with special emphasis on the post-peak softening.
\end{abstract}

\section{INTRODUCTION}

Existence of an inelastic zone, known as the fracture process zone (FPZ), ahead of the macrocrack tip is well documented in literature of concrete fracture. The FPZ is characterized by different toughening mechanisms like microcracking, aggregate bridging, crack deflection which consume considerable part of the energy supplied to the specimen by the external tensile load. The toughening mechanisms occurring in the fracture process zone are responsible for the quasi-brittle behavior of concrete [1, 12]. Several experimental and analytical work have been conducted to explain the toughening mechanisms in the process zone [5, 10, 11]. However, a clear understanding of the role of the different constituents of concrete in its mechanical behavior is still lacking. Plain concrete can be considered as a two phase particulate composite containing coarse aggregates dispersed in mortar matrix. The inherent heterogenous nature of concrete results in its com- plex mechanical behavior. The difference in elastic properties of the coarse aggregates and the surrounding mortar results in making the interface between the two phases relatively weak. When a concrete specimen is subjected to tensile loading, debonding of the aggregates from the mortar matrix occurs and bond cracks intiate at the interface. The bond cracks act as precursors of microcracks and upon increased loading, the microcracks localize to form a macrocrack [2,4]. Extensive debonding can also result in crack deflection [8]. Figure 1] shows schematically the separation of mortar and aggregate at an interface between coarse aggregate and cement matrix.

The mechanism essentially involves different material length scales and a multiscale modeling should be adopted in order to gain a better understanding of the fracture behavior of concrete.

Micromechanics is used to describe the behavior of composite materials wherein the 
macroscopic or overall response of the composite is expressed in terms of the parameters of its constituents. Different homgenization schemes such as the Dilute distribution method, Self consistent method, Mori-Tanaka method etc. exist to relate the microfields of the constituent phases to the macroscopic behavior of the composite. The micromechanics framework provides a robust method and gives an insight to the physics of the different mechanisms occurring at the constituent level of heterogeneous materials. [7, 8] provide a comprehensive account of the various micromechanical models developed in the recent years.

Micromechanics has been used to describe the mechanical behavior for concrete in the past few decades. Pensee et. al. [9] have developed a model coupled with damage mechanics to analyze the behavior of concrete. Damage is accounted for by considering a density of microcracks. Microcrack growth causes anisotropy in the overall behavior. Unilateral effect is also taken into account in case of the microcracks. Mihai and Jefferson [6] used exterior point Eshelby solution to determine the location of microcrack initiation. Constitutive model of concrete has been developed considering a dilute distribution of penny shaped microcracks in the interfacial transition zone (ocurring between the matrix and the aggregate). Stress recovery due to rough closure of the microcracks was developed using contact mechanics.

In the present work, a two dimensional micromechanical model for concrete is proposed to capture the phenomena of aggregate debonding from the surrounding mortar matrix. Airy stress functions are used to determine the stress and displacement fields in the individual phases. The Mori-Tanaka homogenization scheme, which is suitable for materials containing significant percentage of inclusions, is adopted to obtain the overall constitutive behavior of concrete. The effect of debonding on the macroscopic stress-strain behavior of concrete when subjected to monotonic tensile loading is studied.

\section{MICROMECHANICAL MODEL}

Concrete is modeled at meso-scale as a two phase composite containing circular aggregates (phase 'a') dispersed in mortar matrix (phase ' $m$ '). Micromechanics is used to relate the stresses and displacements obtained at the meso-scale to the behavior of concrete as a whole. The elastic properties of the constituents and the volume fractions of the different phases are considered in the formulation. Macroscopic stress and strain are obtained from the corresponding average field quantities of the respective phases [13] as:

$$
\begin{aligned}
\bar{\sigma} & =(1-f) \sigma^{m}+f \sigma^{a} \\
\bar{\epsilon} & =S^{m}: \bar{\sigma}+f\left(S^{a}-S^{m}\right): \sigma^{a} \\
& +f \epsilon^{i n t}
\end{aligned}
$$

where $S^{m}$ and $S^{a}$ are the compliance tensors for the mortar matrix and the aggregate respectively, $f$ represents the volume fraction of aggregates present in the concrete mix, $\sigma^{m} ; \sigma^{a}$ are the average stresses of the individual phases. The term $\epsilon^{i n t}$ accounts for the inelastic strain which occurs at the interface due to displacement discontinuity when debonding of the aggregate from the mortar matrix takes place.

Average stress in the aggregate is given by:

$$
\sigma_{i j}=\frac{1}{A^{a}} \int_{A^{a}} \sigma_{i j} d A
$$

which can be simplified to the form

$$
\sigma_{i j}=\frac{1}{A^{a}} \int_{S^{a}} T_{i} x_{j} d S
$$

The final form of the average stress requires the cumulative effect of the interfacial traction $T_{i}$ acting on the boundary of the aggregate.

The inelastic strain resulting from the displacement jump occurring across the interface is given by:

$$
\epsilon_{\text {int }}=\frac{1}{2 A^{i}} \int_{S}(\|u\| \otimes n+n \otimes\|u\|) d S
$$

where $\|u\|$ is the displacement jump across the interface and $n$ is the normal to the interface.

The Mori-Tanaka (MT) method of homogenization is employed to obtain the relation between the macroscopic quantities and their 
mesoscopic counterparts. In this method, the interaction between the constituents is taken into account by assuming that the inclusion (in this case, the coarse aggregate) is embedded in an infinite matrix and the far field stress (or strain) is approximated by the average matrix stress (or strain) [8].

$$
\begin{aligned}
\bar{\epsilon}= & {\left[S^{m}-f\left\{\left(S^{m}-S^{a}\right) \cdot B\right\}\right.} \\
\cdot & \left.\{(1-f) I+f B\}^{-} 1\right]: \bar{\sigma}+f \epsilon_{\text {int }}
\end{aligned}
$$

where B is the coefficient tensor which relates the average stress components of the aggregates to the applied stress. $\sigma^{a}=B: \sigma^{0}$ relates the average aggregate stress to the far field applied stress. In MT method, the far field stress $\sigma^{0}$ is replaced by $\sigma^{m}$. Equation 6 gives the final result after substitution.

\subsection{Interface behavior}

A traction-separation law showing linear softening is used to describe the behavior of the interface after the interface strength is reached. The area under the softening curve represents the fracture energy $G_{f}$. The cohesive law is characterized by the tensile strength and the shear strength of the interface and the maximum displacement discontinuity (both opening and sliding). Figure 2 shows a schematic representation of the softening behavior of the interface.

\subsection{Stress and displacement fields based on Airy stress functions}

A single aggregate embedded in infinite matrix (mortar) is subjected to far field tensile loading as shown in Figure 3. The coarse aggregate is considered to be circular with radius a. Elastic properties of the two phases (Kolosov constant $\kappa$ and shear modulus $\mu$ ) are distinguished by the superscripts 'a' and 'm'.

The elastic solution to the problem is found with the aid of Airy stress functions. The Airy stress functions for the aggregate phase and the mortar phase are [3]:

$$
\begin{aligned}
\phi^{m} & =\frac{T}{4}\left\{r^{2}-r^{2} \cos (2 \theta)+A a^{2} \log r\right. \\
& \left.+B a^{2} \cos (2 \theta)+C \frac{a^{4}}{r^{2}} \cos (2 \theta)\right\} \\
\phi^{a} & =\frac{T}{4}\left\{D r^{2}+E r^{2} \cos (2 \theta)\right. \\
& \left.+F \frac{a^{4}}{r^{2}} \cos (2 \theta)\right\}
\end{aligned}
$$

The stress and displacement components for both the phases as obtained from the Airy stress functions are:

$$
\begin{aligned}
& \sigma_{r r}^{m}=\frac{T}{4}\left\{2-2 \cos (2 \theta)+A \frac{a^{2}}{r^{2}} \log r\right. \\
& \left.-4 B \frac{a^{2}}{r^{2}} \cos (2 \theta)+6 C \frac{a^{4}}{r^{4}} \cos (2 \theta)\right\} \\
& \sigma_{r \theta}^{m}=\frac{T}{4}\left\{-2 \sin (2 \theta)-2 B \frac{a^{2}}{r^{2}} \sin (2 \theta)\right. \\
& \text { - } \left.6 C \frac{a^{4}}{r^{4}} \sin (2 \theta)\right\} \\
& 2 \mu^{m} u_{r}^{m}=\frac{T}{4}\left\{\left(\kappa^{m}-1\right) r+2 r \cos (2 \theta)-A \frac{a^{2}}{r}\right. \\
& +B\left(\kappa^{m}+1\right) \frac{a^{2}}{r} \cos (2 \theta) \\
& \left.+2 C \frac{a^{4}}{r^{3}} \cos (2 \theta)\right\} \\
& 2 \mu^{m} u_{\theta}^{m}=\frac{T}{4}\{2 r \sin (2 \theta) \\
& \text { - } B\left(\kappa^{m}-1\right) \frac{a^{2}}{r} \sin (2 \theta) \\
& \left.+2 C \frac{a^{4}}{r^{3}} \sin (2 \theta)\right\} \\
& \sigma_{r r}^{a}=\frac{T}{4}\{2 D-2 E \cos (2 \theta)\} \\
& \sigma_{r \theta}^{a}=\frac{T}{4}\left\{2 E \sin (2 \theta)+6 F \frac{a^{2}}{r^{2}} \sin (2 \theta)\right\} \\
& 2 \mu^{a} u_{r}^{a}=\frac{T}{4}\left\{D\left(\kappa^{a}-1\right) r-2 E r \cos (2 \theta)\right. \\
& \left.+F\left(\kappa^{a}-3\right) \frac{a^{3}}{r^{2}} \cos (2 \theta)\right\} \\
& 2 \mu^{a} u_{\theta}^{a}=\frac{T}{4}\{2 \operatorname{Er} \sin (2 \theta) \\
& \left.+F\left(\kappa^{a}+3\right) \frac{a^{3}}{r^{2}} \sin (2 \theta)\right\}
\end{aligned}
$$


The elasticity based solutions are used to obtain the field quantities until the applied loads reach the tensile strength of the interface. The six constants appearing in the expressions are determined by using suitable boundary conditions.

Initially, when the applied load is relatively low, the aggregate is perfectly bonded at the interface. Equilibrium conditions require continuity of stress components $\sigma_{r r}, \sigma_{r \theta}$ and continuity of displacement components $u_{r}, u_{\theta}$ at the interface between the aggregate and the matrix.

$$
\begin{array}{cc}
\left\|\sigma_{r r}(a, \theta)\right\|=0 & \left\|\sigma_{r \theta}(a, \theta)\right\|=0 \\
\left\|u_{r}(a, \theta)\right\|=0 & \left\|u_{\theta}(a, \theta)\right\|=0
\end{array}
$$

where $\|\bullet\|=(\bullet)^{m}-(\bullet)^{a}$ represents the discontinuity at the interface.

Upon increased loading, debonding occurs. For the present analysis it is assumed that the shear strength of the interface is sufficiently high compared to the tensile strength. Hence, only opening (normal direction) of the interface is considered. A trial and error method is adopted for the numerical analysis by assuming some initial value of $\left\|u_{r}\right\|$.

\section{NUMERICAL ANALYSIS}

Numerical analysis is carried out to determine how the properties at the aggregate-mortar scale affect the macroscopic constitutive behavior of concrete. Parameters such as diameter of the coarse aggregates, volume fraction and the elastic properties of the individual phases, behavior of the interface (in terms of tensile strength of the interface and the fracture energy) are considered to have effect on the behavior of concrete.

While the linear elastic behavior is directly obtained from the Airy stress function solution of the boundary value problem; in order to capture the post peak softening behavior of concrete, an incremental iterative procedure is adopted. For the prefectly bonded case, the stress-strain behavior is obtained by incrementing load at each step. However, once the tensile strength of the interface is reached, strain is increased in each step.
In the present study, all aggregates are assumed to have the same diameter. However, a distribution of different aggregate sizes may be assumed to obtain a more realistic concrete mix. Previous studies have shown that the shape of the traction-separation law at the interface has little effect on the behavior of concrete. Therefore, only a linear softening law has been used for analysis for simplicity of the model. Different parameters characterizing the law has been taken into account. The following assumptions have been made for the present analysis:

1. The nonlinear behavior of concrete occurs as a result of aggregate debonding.

2. The aggregate and mortar matrix are isotropic and homogeneous.

3. The volume fraction of aggregates is high in concrete and crack propagation occurs mainly through interface.

4. Aggregates are all of the same size.

5. Plane stress conditions prevail.

6. The shear strength of the interface is greater than its tensile strength. Therefore, in the present study, it is assumed that no slip occurs at the interface in the tangential direction. Separation occurs only in the normal direction.

7. $90 \%$ of the aggregates fail by debonding at the interface [4].

\section{RESULTS}

Analysis in carried out using MATLAB for the different material properties as given in $\mathrm{Ta}-$ ble 1. The results for the effect of different size of aggregates and the volume fraction of aggregates are presented in this section. 
Table 1: Material Properties

\begin{tabular}{cc}
\hline Property & Value \\
\hline $\begin{array}{c}\text { Young's modulus of } \\
\text { coarse aggregate } \mathrm{E}_{\mathrm{a}}\end{array}$ & $70 \mathrm{GPa}$ \\
\hline $\begin{array}{c}\text { Young's modulus of } \\
\text { mortar } \mathrm{E}_{\mathrm{m}}\end{array}$ & $25 \mathrm{GPa}$ \\
\hline $\begin{array}{c}\text { Poisson's ratio of } \\
\text { coarse aggregate } \nu_{\mathrm{a}}\end{array}$ & 0.20 \\
\hline $\begin{array}{c}\text { Poisson's ratio of } \\
\text { mortar } \nu_{\mathrm{m}}\end{array}$ & 0.20 \\
\hline $\begin{array}{c}\text { Tensile strength of } \\
\text { interface } \mathrm{f}_{\mathrm{t}}\end{array}$ & $3.0 \mathrm{MPa}$ \\
\hline $\begin{array}{c}\text { Fracture energy } \\
\mathrm{G}_{\mathrm{fI}}\end{array}$ & $0.03 \mathrm{MPa}-\mathrm{mm}$ \\
\hline
\end{tabular}

Figure 4 shows the macroscopic stress (normalized)-strain behavior for two different aggregate sizes when the volume fraction of aggregates is $45 \%$. For both the sizes, the behavior is similar in the linear elastic regime. The pre-peak behavior is independent of the size of the aggregates. Size of the aggregates enter the formulation only when debonding starts at the interface. The aggregate of smaller size $(D=$ $16 \mathrm{~mm}$ ) debonds at an earlier stage compared to that of the larger aggregate $(D=25 \mathrm{~mm})$. From the area under the stress-strain curve it can be seen that energy dissipated by aggregate of larger size is higher thereby leading to a more ductile behavior after peak load is reached. This can be due to the fact that larger aggregate particles have greater boundary in contact with the matrix. Hence, a higher energy is dissipated when debonding at interface between the aggregate and mortar occurs. To obtain an optimum post-peak softening behavior, a distribution containing aggregates of different sizes should be used.

Figure 5 shows the constitutive behavior of concrete for different volume fractions of aggregate for aggregate size of $20 \mathrm{~mm}$. For higher volume fraction, we can observe a stiffer behavior before the peak stress is reached. This is because, a higher aggregate content increases the stiffness of concrete (aggregate having higher Young's modulus than mortar). At present, no conclusion can be drawn regarding the effect of aggregate volume fraction on the post-peak behavior at the macroscopic scale. Further analysis needs to be conducted until complete failure occurs at the interface.

In order to obtain a smoother response, a refinement is required in the increment of strain (after debonding sets in at the interface) at each step.

\section{CONCLUSIONS}

In the present study, the mechanism of aggregate debonding under uniaxial tension is modeled with the aid of micromechanics. Elastic solution for the field quantities of the individual phases is obtained using Airy stress functions. A linear softening cohesive law is used to characterize the interface. An incremental iterative procedure is adopted for solution when nonlinearity sets in (i.e., the tensile strength of the interface is reached and debonding occurs). The model is able to capture the softening behavior after reaching peak load satisfactorily. Effects of parameters like the aggregate volume fraction and diameter of the aggregate on the overall mechanical behavior of concrete are investigated. This provides valuable insight regarding the behavior of concrete at the constituent level and hence, will enable to design concrete mix for desired performance under tensile loading. The model can be further improved by considering the effect of shear at the interface and a distribution of different sizes of aggregates.

\section{REFERENCES}

[1] Zdenek P Bazant and Jaime Planas. Fracture and size effect in concrete and other quasibrittle materials, volume 16. CRC press, 1997.

[2] Oral Buyukozturk and Brian Hearing. Crack propagation in concrete composites influenced by interface fracture parameters. International Journal of Solids and Structures, 35(31):4055-4066, 1998.

[3] Zhanjun Gao. A circular inclusion with imperfect interface: Eshelbys tensor and 
related problems. Journal of applied mechanics, 62(4):860-866, 1995.

[4] GV Guinea, K El-Sayed, CG Rocco, $\mathrm{M}$ Elices, and $\mathrm{J}$ Planas. The effect of the bond between the matrix and the aggregates on the cracking mechanism and fracture parameters of concrete. $\mathrm{Ce}$ ment and concrete research, 32(12):19611970, 2002.

[5] Victor C Li and Mohamed Maalej. Toughening in cement based composites. part i: cement, mortar, and concrete. Cement and Concrete Composites, 18(4):223237, 1996.

[6] Iulia Carmen Mihai and Anthony Duncan Jefferson. A material model for cementitious composite materials with an exterior point eshelby microcrack initiation criterion. International Journal of Solids and Structures, 48(24):3312-3325, 2011.

[7] Toshio Mura. Micromechanics of defects in solids, volume 3. Springer Science \& Business Media, 2012.

[8] Sia Nemat-Nasser and Muneo Hori. Micromechanics: overall properties of heterogeneous materials. Elsevier, 2013.

[9] Vincent Pensée, Djimedo Kondo, and Luc Dormieux. Micromechanical analysis of anisotropic damage in brittle materials. Journal of Engineering Mechanics, 128(8):889-897, 2002.

[10] E Schlangen and JGMi Van Mier. Experimental and numerical analysis of micromechanisms of fracture of cementbased composites. Cement and concrete composites, 14(2):105-118, 1992.

[11] Surendra P Shah. Toughening mechanisms in quasi-brittle materials, volume 195. Springer Science \& Business Media, 2012.
[12] Surendra P Shah, Stuart E Swartz, and Chengsheng Ouyang. Fracture mechanics of concrete: applications of fracture mechanics to concrete, rock and other quasibrittle materials. John Wiley \& Sons, 1995.

[13] Henry Tan, Young Huang, Cheng Liu, Guruswami Ravichandran, and Glaucio $\mathrm{H}$ Paulino. Constitutive behaviors of composites with interface debonding: the extended mori-tanaka method for uniaxial tension. International Journal of Fracture, 146(3):139-148, 2007. 


\section{FIGURES}

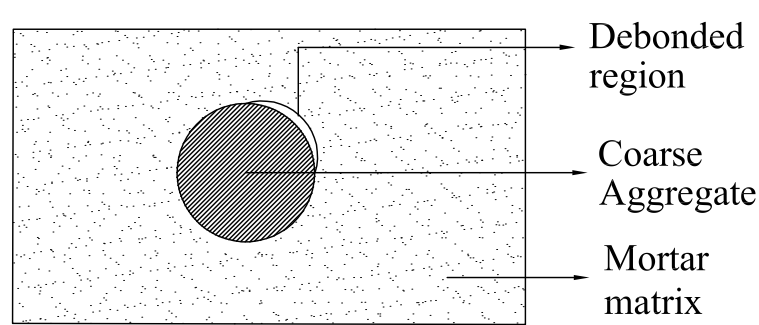

Figure 1: Debonding of aggregate from surrounding matrix

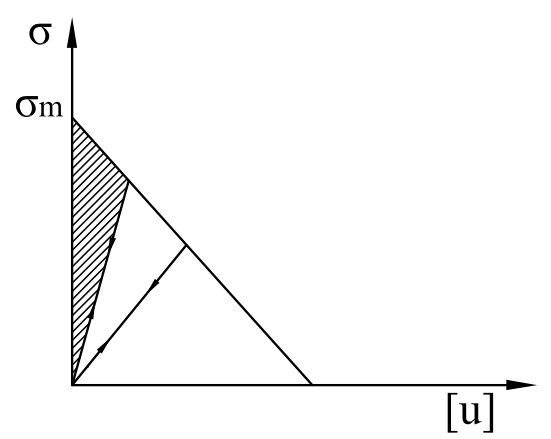

Figure 2: Softening at the interface

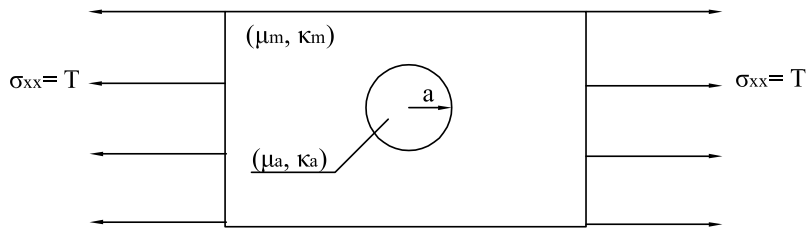

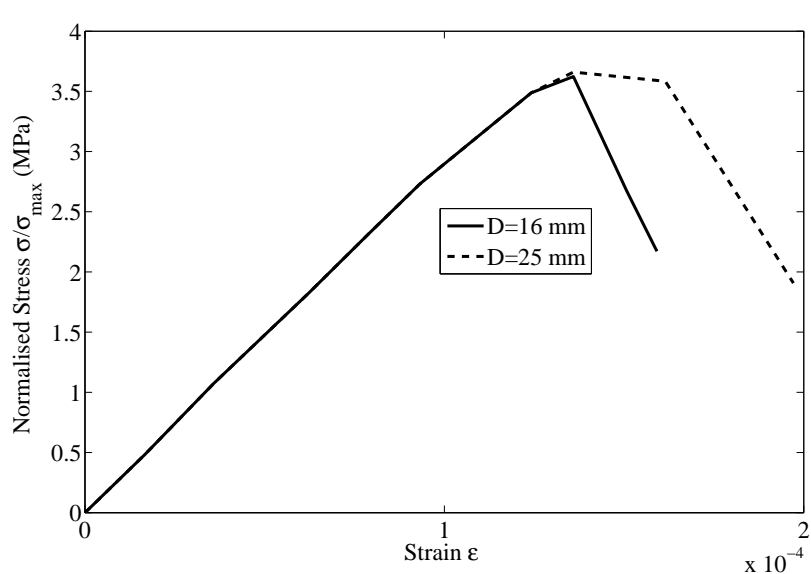

Figure 4: Effect of aggregate diameter

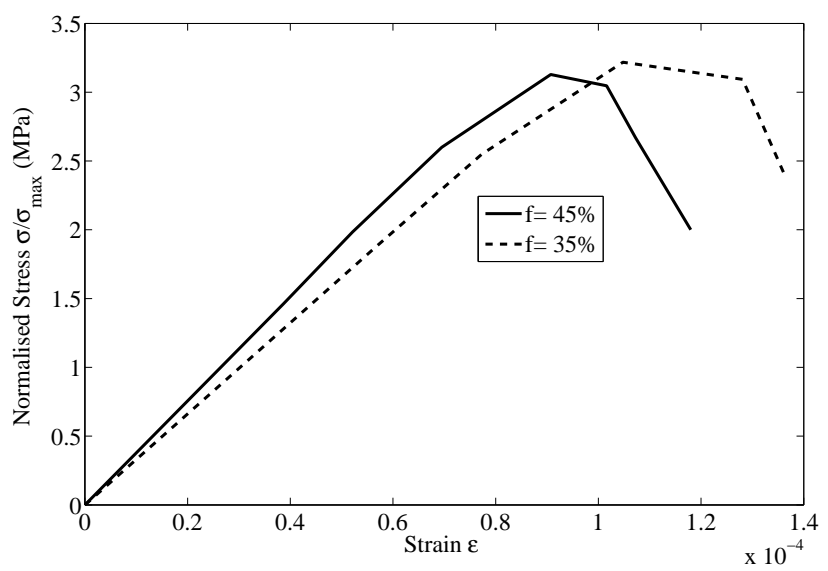

Figure 5: Effect of aggregate volume fraction

Figure 3: Single aggregate subjected to uniaxial tensile loading 